\title{
Una visione dell'Universalienstreit: realismo e nominalismo alla prova della critica
}

Une vision de l'Universalienstreit : réalisme et nominalisme à l'épreuve de la critique

A Vision of the Universalienstreit: Realism and Nominalism to the Test of

Criticism

\section{Andrea Tabarroni}

\section{(2) OpenEdition}

\section{Journals}

Edizione digitale

URL: http://journals.openedition.org/cei/3114

DOI: 10.4000/cei.3114

ISSN: 2260-779X

\section{Editore}

UGA Éditions/Université Grenoble Alpes

\section{Edizione cartacea}

Data di pubblicazione: 30 décembre 2016

Paginazione: 75-84

ISBN: 978-2-84310-337-7

ISSN: 1770-9571

Notizia bibliografica digitale

Andrea Tabarroni, «Una visione dell'Universalienstreit: realismo e nominalismo alla prova della critica», Cahiers d'études italiennes [Online], 23 | 2016, online dal 23 janvier 2017, consultato il 26 mars 2021. URL: http://journals.openedition.org/cei/3114 ; DOI: https://doi.org/10.4000/cei.3114 


\title{
UNA VISIONE DELL'UNIVERSALIENSTREIT: REALISMO E NOMINALISMO ALLA PROVA DELLA CRITICA
}

\author{
Andrea Tabarroni \\ Università di Udine
}

«In ogni opera filosofica o letteraria convivono verità e menzogna, e nel rapporto che intrattengono fra di loro e con le cose, che non arriveremo mai a comprendere fino in fondo, rientra anche il legame profondo fra il

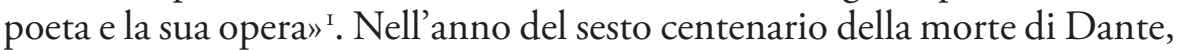
André Jolles dedica su «De Gids» un saggio brillante e impegnato alla visione d'amore nella Vita nuova, in cui si confronta con un tema che gli sta particolarmente a cuore, quello del rapporto tra l'artista e la sua opera come elemento ed espressione di una visione del mondo (delle «cose») che non può tuttavia essere colta "fino in fondo" — perché evidentemente anche nello sforzo ricostruttivo del passato "convivono» sempre "verità e menzogna». Quello della «visione», del resto, è uno dei poli intorno a cui si sviluppa il discorso, che si intreccia in modo indissolubile con l'altro, quello della "forma», in questo caso letteraria, secondo un motivo che già sedici anni prima Jolles aveva introdotto nella sua conferenza friburgense Sull'interpretazione del concetto di verità naturale nelle arti figurative, in cui, sulla scorta di Schiller, troviamo questa dichiarazione: «le arti figurative sembrano ispirarsi, sul piano della forma, a quanto è concretamente visibile nella natura, a prescindere dalle idee astratte che intendono esprimere» ${ }^{2}$.

I. A. Jolles, La visione d'amore nella "Vita nuova", in Id., I travestimenti della letteratura. Saggi critici e teorici (1897-I9I2), S. Contarini (a cura di), premessa di E. Raimondi, Milano, Bruno Mondadori, 2003, pp. 23-43 (qui a pp. 3I-32); per un inquadramento storico-critico si faccia riferimento a S. Contarini, Jolles lettore di Dante. La visione d'amore nella "Vita nuova», "Italianistica», I-2, 2002, pp. 529-539.

2. A. Jolles, Zur Deutung des Begriffes Naturwahrheit in der bildenden Kunst, Freiburg i. B., C. Troemers Universitätsbuchhandlung, 1905, p. 5 (ringrazio S. Contarini per avermi messo a disposizione la sua traduzione ancora inedita, da cui cito). 
Dai Provenzali sino a Dante, secondo il critico olandese, l'idea astratta dell'Amore è al centro dell'elaborazione poetica, dapprima sotto pretesto della sua relazione causale con la visione, poi - attraverso il progressivo affinamento della forma del sonetto - finalmente essa stessa come vivida rappresentazione, che spezza lo schema dell'allegoria per trasformarlo in visione onirica e allo stesso tempo realistica, unità visibile di verità e menzogna. Il problema è dunque quello del realismo nell'arte, in quegli stessi anni oggetto dello scambio intellettuale con Huizinga, ma affrontato, come ha documentato Silvia Contarini, «dal punto di vista del linguaggio delle forme, magari recuperando in un'ottica letteraria le osservazioni di Warburg a proposito della "rivoluzione iconografica" quattrocentesca»" Con Dante Jolles inaugura una nuova fase della sua ricerca morfologica dedicata alle forme letterarie, il cui esito non coinvolge solo, nei primi anni trenta, Forme semplici, ma avrebbe dovuto sfociare anche in un progettato studio autonomo di cui restano solo indicazioni sparse ${ }^{4}$. È proprio nel saggio su Dante del '2I che si trova, in una linea di confronto con l'indagine di Vossler sulla filosofia dello Stilnovo, la chiara rivendicazione di un nuovo spazio epistemologico: «[...] mi sembra che accanto alla prospettiva interpretativa storico-letteraria che si occupa del progresso e della ramificazione della vita del pensiero, si possa considerarne un'altra che mostra lo sviluppo del senso poetico della proporzione e del bisogno di rappresentazione» 5 .

Con accento più icastico, e con riferimento al percorso appena tratteggiato che va dai trovatori ai Siciliani e a Dante, Jolles poi annota: «Se non è privo di significato il fatto che attraverso queste creazioni poetiche si possa pensare Amore, mi sembra tuttavia di minore importanza rispetto al fatto che lo si possa vedere» ${ }^{6}$. Ne risulta che proprio nel primo sonetto

3. S. Contarini, Introduzione ad A. Jolles, I travestimenti della letteratura, cit., p. xxxv. Sul rapporto fra la novella e il ritratto rispetto alla questione del realismo si veda ancora S. Contarini, "Forme artistiche»: Jolles e la teoria della novella, in G. Anselmi et al. (a cura di), Boccaccio e i suoi lettori. Una lunga ricezione, Bologna, Il Mulino, 20I4, pp. I09-I27.

4. Cfr. S. Contarini, «Forme artistiche»: Jolles e la teoria della novella, cit.

5. A. Jolles, La visione d'amore, cit., p. 33. Si confronti in proposito il programma di lavoro di K. Vossler, Die philosophische Grundlagen zum «süssen neuen Stil» des Guido Guinicelli, Guido Cavalcanti und Dante Alighieri, Heidelberg, C. Winter's Universitätsbuchhandlung, I905, pp. I-2: «Nachdem es uns aber, wie ich hoffe, gelungen ist, aus der politischen Dichtung des I3. Jahrhunderts, trotz ihre Spärlichkeit, ein ungefähres Bild vom damaligen Stand des bürgerlichen Gewissens zu formen, darf man erwarten, dass auch die Lage des philosophischen Gewissens in der italienischen Dichtung jener Zeit sich wiedererkennen lasse. Die Quellen fliessen uns sogar viel reichlicher, als es dort der Fall war: denn kaum dürfte eine Dichtung, sei sie lyrischer, didaktischer oder epischer Art, so unbedeutend, so zufällig und nebensächlich erscheinen, dass nicht ein gewisser Tenor und Habitus der Weltanschauung, ein leiser, unbewusster, philosophischer Pulsschlag in ihr lebte».

6. A. Jolles, La visione d'amore, cit., p. 33 (enfasi dell'autore). 
della Vita nuova l'unità di visione e pensiero di Amore contrassegna il superamento dell'allegoria e del simbolismo, segnando il culmine di una forma letteraria e inaugurando una nuova fase, specificamente italiana, nella storia delle «disposizioni mentali» (geestelijke occupaties) di fronte all'opera d'arte'.

Giunto a questo traguardo, tuttavia, Jolles si concede una sorta di malizioso poscritto con cui, ponendosi innanzi a due pagine di Vossler e di Huizinga, apre un'inattesa prospettiva sulla tormentata questione della definizione di Medioevo e Rinascimento come distinti momenti della storia culturale ${ }^{8}$. Il ricorso al registro ironico - segnalato dalla citazione dell'epilogo di un racconto di Oltmans, che strizza l'occhio al lettore di «De Gids» — deve infatti mettere in guardia sul vero intento dell'autore: un modo elegante e leggero per dire "attenzione! In cauda venenum...». Dietro lo schermo del rapporto tra l'arte medievale e il problema degli universali - una questione mai prima affrontata da Jolles, e su cui non tornerà in seguito - , la posta in gioco è ancora quella del realismo artistico e del suo rapporto con l'evoluzione delle forme artistiche nei diversi periodi della storia ${ }^{9}$.

La giustapposizione - «non la contrapposizione», avverte Jolles con velata ironia - di due brani di Vossler e Huizinga, tratti rispettivamente dallo Stilnovo del primo (1904) e dall'Autunno del secondo (I9I8, I $\mathrm{I}^{\mathrm{a}}$ ed.), allestisce un quadro che ricorda quello inscenato sedici anni prima nel Frammento pubblicato su "De Kroniek», in cui la disputa sul carattere tradizionale o progressivo della pittura di Giotto e di van Eyck viene paragonata «alla tenzone tra due cavalieri che da lati diversi colpiscono un bersaglio dal doppio volto, e disputano per decidere se abbia un rivestimento

7. «Le due caratteristiche che ho creduto di ritrovare all'interno della poesia italiana medievale raggiungono qui il loro fine ultimo, confluendo l'uno nell'altra. Il senso del limite e della proporzione unito a un desiderio pressoché illimitato di rappresentazione dà vita a una forma puramente letteraria e indipendente, a un'espressione intensamente visiva dell'Amore. Naturalmente con questo la disputa filosofica non decide per l'una o per l'altra via. L'opera letteraria non rappresenta una soluzione del problema filosofico, ma una forma visibile che ci mette dinanzi agli occhi l'importanza della questione, e contemporaneamente ci soddisfa attraverso la sua realtà estetica liberandoci da ogni contraddizione». (Ibid.)

8. Cfr. S. Contarini, Un dialogo epistolare sul Boccaccio: Jolles, Huizinga e il "problema del Rinascimento", «Studi sul Boccaccio», 39, 20II, pp. 217-24I.

9. A. Jolles, La visione d'amore, cit., p. 40: «Una degna conclusione potrebbe essere quella contenuta in questi versi: "Ma da lontano avverto orecchi / Che dopo l'ultima parola chiedono ancora una parola". Il lettore curioso potrebbe infatti interrogarsi sul rapporto tra questo tipo di amore e le correnti del pensiero medievale, fino al realismo e al nominalismo, e magari, visto che il dio porta un nome antico, risalire fino all'Antichità e ai suoi rapporti con quello che sarà il Rinascimento...» I versi sono tratti, come segnala S. Contarini (ibid., n. I5) da un racconto di J. F. Oltmans pubblicato nel I840 sul quarto numero della principale rivista letteraria olandese, di cui fu anche redattore (J. F. Oltmans, Het Testament, «De Gids», 4, I840, pp. 349-380). 
d'oro o d'argento»" ${ }^{\text {IO }}$. Il bersaglio dei cavalieri è invece ora la relazione tra arte medievale e Universalienstreit. Vossler vi si rivolge riconducendo il realismo degli universali a un'istanza di razionalizzazione e spiegazione dei fenomeni, che in fine vengono trascurati «per cercare il valore principale nel mondo ideale della ragione e nell'universale», secondo quella che «è nella sua essenza più intima una visione scientifica e dunque non artistica, non estetica» ${ }^{\mathrm{II}}$. Il realismo degli universali conduce dunque all'antirealismo estetico; simbolismo e allegoria nell'arte medievale, in una prospettiva già molto vicina a quella di Croce, sono un ostacolo alla poesia, e Vossler, nel seguito immediato al brano riportato da Jolles, giunge sino a sottoscrivere il vecchio giudizio che vede nell'Inferno il vertice dell'arte dantesca, che s'indebolisce man mano che sale al cielo ${ }^{\mathrm{I2}}$.

Anche Huizinga, d'altra parte, subito prima dell'inizio del brano citato da Jolles, riconosce «il legame indissolubile tra il simbolismo e il "realismo" nel senso medievale della parola» e lo collega al «modo di pensare primitivo» e all'antropomorfismo. E tuttavia, proprio al contrario di Vossler, per Huizinga il realismo simbolista degli universali porta con sé il sentimento mistico, "presta a tutte le rappresentazioni un più alto valore estetico ed etico", "è come la musica eseguita sul testo delle dottrine logicamente formulate» ${ }^{13}$.

A questo punto tuttavia, una volta che si sia ampliato lo sguardo del lettore di là dalla sineddoche dei testi citati da Jolles, appare chiaro che

Io. Il contesto e la citazione di A. Jolles, Fragment, "De Kroniek», 1905, pp. 300-302, sono ricavati da S. Contarini, Introduzione ad A. Jolles, I travestimenti della letteratura, cit., pp. XXviII-XXIX.

II. Cfr. K. Vossler, Die philosophischen Grundlagen, cit., p. 2I (mi valgo qui della traduzione del brano fornita da S. Contarini in A. Jolles, I travestimenti della letteratura, cit., p. 40).

I2. Così prosegue infatti K. Vossler, Die philosophischen Grundlagen, cit., p. 2I: «und der grösste Dichter des Mittelalters, der das Unglück hatte, im Bann dieser antiästethischen Weltanschauung zu stehen, konnte sich darum unbehindert nur in der negativen Welt des Unwesentlichen und Nichtseinsollenden, in der Welt der Verdammnis bewegen. Seine wahre dichterische Heimat hat Dante in der Hölle gefunden. Es ergeht ihm wie dem erdgeborenen Riesen: in dem Masse wie er sich deem Himmel nähert, erstickt in ihm der Dichter».

13. Cito dalla traduzione italiana della seconda edizione di J. Huizinga, Autunno del Medioevo, introduzione di E. Garin, Milano, BUR, 2004 ( $\mathrm{I}^{\mathrm{a}}$ ed. italiana Firenze, Sansoni, I940), pp. $285-286$ (ma occorre tener presente che nel I92I Jolles conosceva il testo nella prima edizione del I9I9, che, come avverte S. Contarini, subirà modifiche anche strutturali accogliendo suggerimenti dello stesso Jolles): "[...] ciò che importa innanzi tutto non è la disputa di sottili teologi, ma sono le idee che dominano tutta la vita della fantasia e del pensiero, quale si manifesta nell'arte, nella moralità, nella vita di ogni giorno. Queste idee sono di un realismo estremo, non per il fatto che l'alta teologia si era formata alla scuola del neoplatonismo, bensì perché il realismo, indipendentemente da ogni filosofia, era il modo di pensare primitivo. Per lo spirito primitivo tutto ciò che può essere nominato assume subito un essere, anche se si tratta di qualità, di concetti o di qualcosa di simile. Tutto ciò si proietta immediatamente ed automaticamente sul cielo. Il loro essere può quasi sempre (benché non sia sempre necessario) venir concepito come un essere personale: in ogni momento si può iniziare la ridda dei concetti antropomorfici. Ogni realismo in senso medievale è in ultima analisi un antropomorfismo. Quando il pensiero, che ha riconosciuto all'idea una realtà indipendente, vuole tradursi in immagini, non lo può fare che col mezzo della personificazione. Ecco il trapasso dal simbolismo e dal realismo all'allegoria». 
i due cavalieri, come nella vecchia analogia proposta in «De Kroniek», giacciono entrambi «stesi a terra feriti a morte», e il buffone che passa di lì può mostrare loro che "entrambi e nessuno dei due avevano ragione». Realismo e nominalismo non si affrontano nella vita dell'arte come due antitesi irriducibili, entrambi sono compresenti nello spirito primitivo, entrambi concorrono a far incontrare immagine e pensiero, i sensi con la ragione, e proprio l'arte italiana del Duecento lo dimostra, «con Dante come capostipite e come punto d'arrivo» ${ }^{14}$. Il colpo finale è portato da Jolles con ironica grazia, rivendicando pienamente a Dante, il «poeta della visione d'Amore», proprio le parole con cui Anselmo aveva bollato, due secoli prima della Vita nuova, i suoi avversari nominalisti come «dialettici eretici». Senza curarsi di citare la fonte, Jolles riporta il latino dell'arcivescovo di Canterbury:

in eorum quippe animabus ratio, quae et princeps et judex omnium debet esse quae sunt in homine, sic est imaginationibus corporalibus obvoluta, ut ex eis se non possit evolvere [nelle loro anime la ragione, che deve essere signore e giudice di tutto quello che è nell'uomo, è talmente avviluppata dalle immaginazioni del corpo, da non potersene districare].

A misurare appieno le implicazioni di questo giudizio con cui Dante viene provocatoriamente rivendicato al nominalismo estetico vale la pena di ricostruire, seppure a grandi linee, la fortuna per certi versi emblematica di questo brano ${ }^{15}$. Jolles cita dall'Epistula de incarnatione Verbi et de

I4. A. Jolles, La visione d'amore, cit., pp. 4I-42: «Mi sembra che il significato profondo della disputa non possa essere colto prospettando una rigida opposizione che esclude l'uno o l'altro concetto. Nello spirito primitivo le due correnti esistono l'una accanto all'altra, rappresentano sia l'esigenza di far passare la realtà sensibile delle percezioni al vaglio della ragione, sia quello di ricondurre la ragione alla dimensione del sensibile. La scolastica percepisce questa ambivalenza e compie uno sforzo enorme per risolvere la controversia in una delle due direzioni, ma tale tentativo teorico dimentica spesso il contatto con la realtà e la dimensione della pratica. Gli individui e l'arte viceversa non sono ancora pronti né disposti a scegliere per l'una o per l'altra. L'arte italiana del Duecento - come si può decidere se per Giotto gli universalia venivano prima o dopo le cose? — con Dante come capostipite e come punto d'arrivo, rimane una testimonianza eloquente di tale duplicità intrinseca».

I5. Per una valutazione della tradizione di studi rapidamente ripercorsa in quanto segue cfr. J. Jolivet, Les études de philosophie médiévale en France de Victor Cousin à Étienne Gilson, in R. Imbach, A. Maierù (a cura di), Gli studi di filosofia medievale fra Otto e Novecento. Contributo a un bilancio storiografico, Atti del Convegno internazionale di Roma (2I-23 settembre 1989), Roma, Edizioni di Storia e Letteratura, I99I, pp. I-20; W. J. Courtenay, In Search of Nominalism: Two Centuries of Historical Debate, ivi, pp. 233-25I (pubblicato anche in Id., Ockham and Ockhamism. Studies in the Dissemination and Impact of His Thought, Leiden, Boston, Brill, 2008, pp. I-I9); G. Piaia, La «svolta francese» (I800-I820) nell'approccio alla filosofia medievale, in J. Meirinhos, O. Weijers (a cura di), Florilegium Mediaevale. Études offertes à Jacqueline Hamesse à l'occasion de son éméritat, Louvain-la-Neuve, Fédération internationale des instituts d'études médiévales, 2009, pp. 45I-467. Per un'informazione approfondita e aggiornata sull'intreccio delle dottrine e sulla posta in gioco nel XII secolo intorno alla questione degli universali ci si può ora utilmente rivolgere a Ch. Erismann, L'Homme commun. La genèse du réalisme ontologique durant le haut Moyen Âge, Paris, Vrin, 20II, e C. Tarlazzi, Individui universali. Il realismo di Gualtiero di Mortagne nel XII secolo, Barcelona, Roma, Fédération internationale des instituts d'études médiévales, 2016. 
fide Trinitatis, scritta da Anselmo a papa Urbano II intorno al Io94 con l'intento di denunciare le pericolose dottrine teologiche di Roscellino di Compiègne ${ }^{16}$. Messo in allarme da quanto gli viene riferito da fonti degne di fede, ma con l'obbiettivo soprattutto di riaffermare la competenza vescovile sull'insegnamento della dialettica oltre che della teologia, Anselmo ribadisce con forza il proprio motto caratteristico, secondo cui senza la fede non si può avere reale comprensione, e ne trae la necessità di escludere dalle dispute teologiche coloro che non mostrano di avere sufficiente maturità e di avere dato prova della loro ortodossia. A riprova presenta al pontefice il caso del maestro Roscellino, che afferma che «le sostanze universali non sono che flatus vocis» e la cui facoltà razionale è, appunto, "talmente avviluppata nelle immaginazioni del corpo, da non potersene districare ed estrarne quelle realtà che essa dovrebbe contemplare separate e pure» ${ }^{17}$.

Sin dal pionieristico lavoro di Victor Cousin sulle opere inedite di Abelardo (1836), che inaugura si può dire la moderna storiografia filosofica medievale, questa testimonianza è assurta a livello di emblema del contrasto tra realismo e nominalismo, colto nel momento stesso della sua nascita, nonostante si tratti ovviamente di una testimonianza di parte (Anselmo è un campione del realismo), non corroborata peraltro da documenti originali: Roscellino non ha lasciato infatti scritti dottrinali. Sotto l'influenza della filosofia britannica del diciottesimo secolo — da cui Cousin intendeva distanziare il concettualismo di Abelardo, considerato

I6. Per le due redazioni della lettera l'edizione di riferimento è ora Anselmo d'Aosta, Epistula de incarnatione Verbi, in S. Anselmi Cantuariensis Archiepiscopi Opera Omnia, ed. F. S. Schmitt, Edinburgh, F. Nelson and Sons, I946, vol. I, pp. 28I-290 (prior recensio) e vol. 2, pp. 3-35.

17. Cfr. Anselmo d'Aosta, Epistula de incarnatione Verbi, c. I, in S. Anselmi, cit., vol. 2, p. 9, 11. 2I-22 (poco dopo aver ribadito lo stilema agostiniano ricalcato su $I s .7,9$ «nisi credideritis, non intelligetis»): «illi utique nostri temporis dialectici, immo dialecticae haeretici, qui non nisi flatum vocis putant universales esse substantias», e p. Io, 11. I-2: «in eorum quippe animabus ratio, quae et princeps et judex omnium debet esse quae sunt in homine, sic est in imaginationibus corporalibus obvoluta, ut ex eis se non possit evolvere, nec ab ipsis ea quae ipsa sola et pura contemplari debet, valeat discernere». Sul confronto tra Anselmo e Roscellino si vedano in part. le ricerche di C. Mews, St Anselm and Roscelin: Some New Texts and Their Implications. I. The De incarnatione Verbi and the Disputatio inter christianum et gentilem, "Archives d'histoire doctrinale et littéraire du Moyen Âge», 58, 1991, pp. 55-98; Id., Nominalism and Theology before Abaelard: New Light on Roscelin of Compiègne, "Vivarium", 30, 1992, pp. 4-34; Id., St Anselm, Roscelin and the See of Beauvais, in Anselm. Aosta, Bec and Canterbury, Papers in Commemoration of the Nine-Hundredth Anniversary of Anselm's Enthronement as Archbishop, 25 September I093, D. E. Luscombe, G. R. Evans (a cura di), Sheffield, Sheffield Academic Press, 1996, pp. I06-199; Id., The Trinitarian Doctrine of Roscelin of Compiègne and Its Influence: Twelfth-century Nominalism and Theology Reconsidered, in Langages et philosophie. Hommage à Jean Jolivet, A. de Libera, A. Elamrani-Jamal, A. Galonnier (a cura di), Paris, Vrin, 1997, pp. 347-364; Id., St Anselm and Roscelin of Compiègne: Some New Texts and Their Implications. II. A Vocalist Essay on the Trinity and Intellectual Debate c. I080-II20, "Archives d'histoire doctrinale et littéraire du Moyen Âge», 65, I998, pp. 39-90 (questi cinque articoli sono stati ripubblicati in Id., Reason and Belief in the Age of Roscelin and Abelard, Aldershot, Ashgate, 2002, VI-X). 
fondatore della tradizione francese - , dalla testimonianza di Anselmo fu subito tratta una visione del nominalismo come una sorta di empirismo, anzi di sensismo: imprigionato dalle sensazioni il nominalista non è in grado di vedere gli oggetti astratti e universali ${ }^{18}$. In verità l'accusa di Anselmo va meglio intesa da un punto di vista neoplatonico, come richiamo alla necessità per la ragione di elevarsi al di sopra della sola considerazione dei corpi, che non produce vero pensiero, ma semplici immagini.

Come un sensista Roscellino viene infatti dipinto da Xavier Rousselot nel I840, proprio sulla base della testimonianza di Anselmo, e considerato iniziatore di quella corrente del pensiero medievale che giunge sino a Ockham e da cui in seguito trarranno ispirazione Locke e Malebranche ${ }^{19}$. Lo stesso ritratto si incontra nelle pagine dedicate a Roscellino da Charles de Rémusat nel suo Abelardo (I845) e anche nell' Histoire de la philosophie scholastique di Barthélemy Hauréau (I876), forse la migliore trattazione storica sull'argomento nel XIX secolo, in cui si trova una difesa di Roscellino improntata al pensiero di Reid e di Rousseau; ancora una volta il nominalista estremo, impersonato dal monaco di Compiègne, viene raffigurato come un rappresentante del senso comune, alieno a dar credito a ciò che non si può vedere né toccare ${ }^{20}$. Alla fine del secolo, nel i896, François Picavet dedica ancor maggiore applicazione, nel suo Roscelin, alla rivalutazione del protonominalista, difendendolo dalle accuse di eresia e

I8. Cfr. V. Cousin, Introduction a Ouvrages inédits d'Abélard pour servir à l'histoire de la philosophie scolastique en France, Paris, Imprimerie Royale, I836, p. Lxxxix (la citazione di Anselmo), e si veda anche il giudizio a p. c: «Roscelin est le précurseur de l'école empirique. Sans doute cette école est bien faible encore dans Roscelin, mais elle commence avec lui pour ne plus finir". Petri Abaelardi opera hactenus seorsim edita nunc primum in unum collegit [...] Victor Cousin adjuvantibus C. Jourdain et E. Despois, 2 voll., Paris, Durand, I849; V. Cousin, Fragments philosophiques. Philosophie scholastique, $2^{\mathrm{a}}$ ed., Paris, Ladrange, I84O, pp. I23, I38.

19. Cfr. X. Rousselot, Études sur la philosophie dans le Moyen-Âge. Première partie, comprenant les deux premières époques, Paris, Joubert, I840, pp. I53-I57. Per la stretta connessione stabilita da Rousselot tra il nominalismo medievale e le posizioni moderne dell'empirismo e del sensismo cfr. W. Courtenay, In Search of Nominalism, cit., n. 8.

20. Cfr. Ch. de Rémusat, Abélard, 2 voll., Paris, Ladrange, I845, vol. I, pp. 505-506 (giudizio sul nominalismo come forma di «rigido sensualismo»); B. Hauréau, De la philosophie scolastique, Mémoire couronné par l'Académie des Sciences morales et politiques, 2 voll., Paris, Pagnerre, I850; Id., Histoire de la philosophie scolastique. Première partie (de Charlemagne à la fin du XII ${ }^{\mathrm{e}}$ siècle), Paris, Durand et Pedone Lauriel, I872, p. 250 (citazione di Anselmo) e pp. 256-257: «Il dit donc [...] qu’une substance est une nature de laquelle on ne peut rien retrancher, rien distraire, sans l'anéantir. C'est le langage du sens commun, de la vraie philosophie, d'Aristote et, hâtons-nous de le dire, de Platon lui-même». Id., Notices et extraits de quelques manuscrits latins de la Bibliothèque nationale, vol. 5, Paris, Klincksieck, I892, pp. 290-338. Di notevole interesse, a testimoniare l'influenza di Reid e di Rousseau sul grande storico francese della filosofia medievale, la notizia sulla teoria medievale delle «idee-immagini», ripubblicata con ottima cura in B. Hauréau, A. Grondeux, Idées-images (1857-I86I), "Journal des savants», 2, I, 2003, pp. 393-4I5 (nell'ambito del numero tematico su "Barthélémy Hauréau [I8I2-I896], le Bénédictin de la République, Actes de la journée d'étude du 9 mars 2002 à l'Institut de France»). 
dalle imputazioni filosofiche dei suoi detrattori (tra cui lo stesso Abelardo), ma non muta il quadro sostanziale delle sue dottrine ${ }^{21}$.

Lo stesso schema troviamo, quasi sempre sorretto dal riferimento alla testimonianza anselmiana, anche nelle trattazioni ottocentesche su Roscellino in lingua tedesca. I principali manuali della seconda metà del secolo — da Kaulich a Stöckl alle prime edizioni dello Überweg — si fondano principalmente sui lavori di Rémusat e di Hauréau ${ }^{22}$. Dalla medesima base informativa traggono alimento anche le trattazioni dedicate dal pastore evangelico Köhler a Realismus und Nominalismus (I858) e da Karl Barach alla Geschichte der Nominalismus vor Roscellin (I866) ${ }^{23}$. Del tutto caratteristica in questo periodo è la conclusione del paragrafo su Roscellino contenuta nel saggio di Johann Heinrich Löwe su Der Kampf zwischen dem Realismus und Nominalismus im Mittelalter (I876):

Come accade in ogni epoca, così anche il nominalismo di Roscellino non poté evitare di ricadere nel sensualismo. Se infatti soltanto la cosa individuale è un vero ente, allora, dato che questa può essere percepita solo dai sensi, non vi può essere altra fonte né altro ambito di applicazione per la conoscenza se non la percezione sensibile ${ }^{24}$.

A suggello di tale giudizio viene naturalmente riportata la consueta testimonianza di Anselmo sull'incapacità del nominalista di liberarsi dai lacci dell'immaginazione corporea.

2I. Cfr. F. Picavet, Roscelin philosophe et théologien d'après la légende et d'après l'histoire. Sa place dans l'histoire générale et comparée des philosophies médiévales, Paris, Alcan, I9II (revisione ampliata della precedente edizione del I896).

22. Cfr. W. Kaulich, Geschichte der scholastischen Philosophie. I. Theil. Entwicklung der scholastischen Philosophie von Johannes Scotus Eriugena bis Abälard, Prag, F. Tempsky, I863, pp. 265-277; A. Stöckl, Geschichte der Philosophie des Mittelalters. Erster Band. Periode der Entstehung und allmähligen Ausbildung der Scholastik, Mainz, Kirchheim, I864, pp. I35-I40; F. Überweg, Grundriss der Geschichte der Philosophie von Thales bis auf die Gegenwart. Zweiter Theil. Die mittlere oder die patristische und scholastische Zeit, Dritte... Auflage, Berlin, S. Mittler und Sohn, I868, pp. II8-I23, ma già a partire dalla settima edizione, postuma, curata da M. Heinze, Berlin, S. Mittler und Sohn, I868, compare il seguente giudizio (p. I50): «Wie mit dem Nominalismus überhaupt der Sensualismus verbunden zu sein pflegt, so auch bei Roscellin». Si vedano inoltre H. Ritter, Geschichte der christlichen Philosophie, III, Hamburg, Perthes, I844, pp. 354-474; C. Ubaghs, Du problème ontologique des universaux et de la véritable signification du réalisme, Louvain, Vanlinthout, I86I; C. Prantl, Geschichte der Logik im Abendlande, Leipzig, Hirzel, I86I, vol. II, pp. II6-I52 (2 $2^{a}$ ed., Leipzig, I885).

23. Cfr. H. O. Köhler, Realismus und Nominalismus in ihrem Einfluss auf die dogmatischen Systems des Mittelalters. Ein Beitrag zur Dogmengeschichte und zur Geschichte der Philosophie, Gotha, F. A. Perthes, I858, pp. 33-42; K. Z. Barach, Zur Geschichte des Nominalismus vor Roscellin. Nach bisher unbenützten handschriftlichen Quellen der Wiener Kaiserlichen Hofbibliothek, Wien, Braumüller, I866.

24. J. H. Löwe, Der Kampf zwischen dem Realismus und Nominalismus in Mittelalter. Sein Ursprung und sein Verlauf, Oarg, Kosmack und Neugebauer, 1876, p. 45 (subito di seguito viene ancora una volta riportata la citazione di Anselmo). E si vedano ancora J. Reiners, Der aristotelische Realismus in der Frühscholastik. Inaugural-Dissertation zur Erlangung der Doktorwürde genehmigt von der philosophischen Fakultät der Rheinischen Friedrich-Wilhelms-Universität zu Bonn, Bonn, Trapp, 1907; Id., Der Nominalismus in der Frühscholastik, Münster, Aschendorff, I9Iо (BGPTM, 8); H. Dehove, Qui praecipui fuerint labente XII saeculo ante introductam Arabum philosophiam temperati realismi antecessores, Insulis, Lefebvre-Ducrocq, 1908. 
Mi sia concessa a questo punto una minima annotazione di carattere filologico. La versione del testo anselmiano citata da Jolles esibisce una piccola lezione variante rispetto al textus receptus, immancabilmente accolto non solo nella versione, per così dire, archetipica di Cousin, ma anche nella Patrologia del Migne, e di conseguenza in tutti gli autori che ho citato sinora (l'edizione critica dello Schmidt era ancora di là da venire). Secondo la versione comunemente diffusa (ancor oggi confermata), Anselmo afferma che la mente dei suoi avversari nominalisti non riesce a elevarsi alla contemplazione degli universali poiché a tal punto "est in imaginationibus corporalibus obvoluta», mentre nel testo letto da Jolles lo «in» non compare. La variante non altera sensibilmente il significato del brano, e si potrebbe senz'altro facilmente spiegare in modo meccanico, per un refuso tipografico o per lieve disattenzione di Jolles medesimo. Se si rinvenisse, ciò che sinora non mi è accaduto, una fonte plausibile della citazione anselmiana in cui si trovi la medesima omissione, la coincidenza in errore costituirebbe a mio avviso in questo caso un forte indizio per stabilire un rapporto di dipendenza letteraria ${ }^{25}$. Ma non mi sentirei neppure di escludere apriori un'ulteriore possibilità, quella cioè che Jolles stesso sia intervenuto, con tocco minimo e sapiente, a meglio accordare Anselmo ai suoi scopi: nell'applicare a Dante l'identikit tratteggiato per Roscellino, un conto è infatti suggerire l'idea che il poeta della visione d'Amore non potesse staccarsi da una situazione in cui le immagini dei corpi sono dominanti, altra cosa è invece affermare che egli non può sottrarsi del tutto all'azione di quelle immagini, che è quanto dire alla loro visibilità.

Sia quel che sia della questione filologica, che in fondo interessa relativamente, è certo che Jolles intende sottolineare l'indissolubilità del nesso tra immagine e idea, o forse meglio tra immagine e forma, visibilità e struttura, in un modo che ricorda il tentativo di chi in quegli stessi anni, in diverso ambito, andava cercando di sviluppare un ulteriore sistema di morfologia dinamica, come Carl Gustav Jung. Nello stesso I92I in cui Jolles si occupa della visione d'Amore in Dante, Jung pubblica i suoi Tipi psicologici, in cui si può leggere una lunga e impegnata trattazione

25. In effetti la medesima omissione si riscontra nel testo della citazione riportato da W. Kaulich, Geschichte der scholastischen Philosophie, cit., p. 270, in nota, ma la dipendenza è qui esclusa dalla presenza di un'ulteriore variante rispetto al testo di Jolles: «in eorum quippe animabus ratio, quae [et aggiunge Jolles in accordo con il textus receptus] princeps et judex omnium debet esse quae sunt in homine, sic est imaginationibus corporalibus obvoluta, ut ex eis se non possit evolvere»; Kaulich cita dall'edizione maurina di G. Gerberon, Sancti Anselmi ex Beccensi Abbate Cantuariensis Archiepiscopi Opera, necnon Eadmeri monachi Cantuariensis Historia Novorum et alia opuscula, Lutetie Parisiorum, L. Billaine et J. Du Puis, I675, p. 43. 
storico-evolutiva del confronto tra nominalismo e realismo ${ }^{26}$. Anche qui, come in Huizinga, si ritrova il nesso tra realismo filosofico e «mentalità primitiva ${ }^{27}$. Ma l'approccio si avvicina piuttosto a quello di Jolles quando si legge che, se considerata unicamente dal punto di vista logico e intellettuale, l' «antitesi fra nominalismo e realismo [...] deve necessariamente sboccare nel paradosso», quindi, per dirla con il «buffone» di Jolles, nella situazione in cui entrambi i cavalieri giacciono a terra, feriti a morte, entrambi vincitori e sconfitti ${ }^{28}$. La soluzione del conflitto diviene possibile soltanto assumendo «un terzo punto di vista mediatore», quello fornito, secondo Jung, dall' "attività specifica della psiche», la fantasia, che «è a un tempo sentimento e pensiero, è intuizione e sensazione. Non v'è funzione psichica che in essa non si trovi indistinguibilmente connessa con le altre funzioni psichiche. Essa appare talora come cosa primordiale, talora come ultimo e massimo prodotto della sintesi di ogni facoltà ${ }^{29}$.

In fondo non manca che un passo per scorgere nella «fantasia» di Jung l'organo produttore delle "forme artistiche» indagate da Jolles, in cui l'attività creativa dell'uomo tenta di ricomporre di volta in volta, nelle varie epoche, «il senso del limite e della proporzione» («Belle Proportion») con «il desiderio pressoché illimitato di rappresentazione» ("Besoin Visuel»), secondo l'allegoria dolcemente parodica con cui si conclude il saggio sulla visione d'Amore in Dante ${ }^{30}$.

26. Cfr. C. G. Jung, Psychologische Typen, Zürich, Rascher, I92I, trad. it. di C. Musatti, L. Aurigemma, Torino, Bollati Boringhieri, 1969, pp. 37-74.

27. Ivi, p. 4I: «Nei primitivi, infatti, l'Imago, la risonanza psichica della percezione sensoriale, è così intensa e così fortemente intrisa di sensorialità che quando si affaccia riprodotta, e cioè come immagine mnemonica spontanea, può assumere talvolta persino il carattere di allucinazione. [...] Noi ci limitiamo a "pensare" ai morti, il primitivo, invece, li percepisce appunto per la straordinaria materialità delle immagini della sua mente. Di qui deriva la credenza negli spiriti dei primitivi. Gli spiriti sono ciò che noi chiamiamo semplicemente pensieri. Il primitivo, quando "pensa", ha effettivamente delle visioni la cui realtà concreta è tale che egli scambia costantemente ciò che è psichico con ciò che è reale».

28. Ivi, p. 62. E Jung spiega poco dopo: «Ogni formulazione logico-intellettuale, per quanto perfetta sia, annulla la vivacità e l'immediatezza dell'impressione provocata dall'oggetto. Essa deve annullarla per poter appunto pervenire a formularla. Ma con ciò va perduto proprio quanto sembra essere assolutamente essenziale all'atteggiamento estroverso, cioè il riferimento all'oggetto reale. Non sussiste perciò alcuna possibilità di trovare, attraverso l'uno o l'altro atteggiamento, una qualsiasi formula unificatrice che sia soddisfacente». Si confronti con quanto afferma Jolles nel brano riportato sopra in n. 7 .

29. Ivi, p. 63.

30. Con questo non intendo naturalmente avanzare l'ipotesi di un rapporto diretto, personale o intertestuale, tra Jolles e Jung, quanto piuttosto suggerire che anche queste due figure di «irregolari» possono opportunamente inserirsi nel paradigma culturale che in Italia è stato ben delineato, ad esempio, dal saggio di A. Pinotti, Il corpo dello stile. Storia dell'arte come storia dello stile a partire da Semper, Riegl, Wölffin, Milano, Mimesis, $200 \mathrm{I}$. 